\title{
Selective impairment in recognizing the familiarity of self faces in schizophrenia
}

\author{
${\text { ZHANG } \text { Lin }^{1 \dagger}, \text { ZHU Hong }^{2 \dagger}, \text { XU Miao }}^{1}$, JIA HongXiao ${ }^{1,2^{*}} \&$ LIU Jia ${ }^{1,3^{*}}$ \\ ${ }^{1}$ State Key Laboratory of Cognitive Neuroscience and Learning, Beijing Normal University, Beijing 100875, China; \\ ${ }^{2}$ Beijing Anding Hospital, Capital Medical University, Beijing 100088, China; \\ ${ }^{3}$ Graduate University of the Chinese Academy of Sciences, Beijing 100049, China
}

Received December 1, 2011; accepted February 13, 2012

\begin{abstract}
The concept of self is a fundamental characteristic of the human mind, and the alteration of self is thought to be a core deficit of schizophrenia. Previous studies have demonstrated that patients with schizophrenia are deficient in self-face recognition. Because self faces are not only self-related but also highly familiar, it is unclear whether such deficit arises from the breakdown of the self-awareness or the failure of recognizing the familiarity of self faces. Here we directly tested these two alternatives by instructing patients with schizophrenia to recognize the identity of a morphed face created by blending face features between any of two identities from the self face, a familiar face, and a novel face. We found that there was no association between the recognition of the self and the recognition of the familiarity, suggesting these two component processes are independent in schizophrenia. Further, patients with schizophrenia were significantly worse in recognizing the familiarity of faces than normal participants, whereas no difference in the sense of self was found between the two groups. Taken together, our finding suggests that it is the sense of familiarity, not the sense of self, that is selectively impaired in self-face recognition in schizophrenia. Thus, our study challenges the hypothesis that the deficit in self-face recognition in schizophrenia reflects the breakdown of self-awareness.
\end{abstract}

schizophrenia, self-face recognition, familiarity, self-awareness, face processing

Citation: Zhang L, Zhu H, Xu M, et al. Selective impairment in recognizing the familiarity of self faces in schizophrenia. Chin Sci Bull, 2012, 57: 1818-1823, doi: $10.1007 / \mathrm{s} 11434-012-5109-\mathrm{Z}$

Animals can recognize faces, but only humans and great apes can recognize their own faces [1-3]. Consistent with this observation, self-face recognition is associated with distinct patterns of behavioral performance [4-7] and neural activation [8-11] as compared with the recognition of others' faces. Further, numerous studies have reported that patients with schizophrenia [12,13] and normal individuals with schizotypal personality [14-16] are impaired in self-face recognition. Because self faces are not only highly familiar [17] but also self-related [18], two related but distinct theories have been proposed. One interpretation is that the poor performance reflects deficits in recognizing the familiarity of self faces (i.e., a deficit in the sense of famili-

\footnotetext{
† These authors contributed equally to this work.

* Corresponding authors (email: jhxlj@vip.163.com; liujia@bnu.edu.cn)
}

arity) $[19,20]$, whereas the other argues that the disrupted behavior arises from the breakdown of self-awareness in schizophrenia (i.e., a deficit in the sense of self) $[13,16]$. To test these two alternatives, we adopted a paradigm to exclude the effect of general cognitive ability and to decouple self-face recognition into two component processes, the sense of familiarity and the sense of self. We then directly examined which component process of self-face recognition is disrupted in schizophrenia.

Previous studies have shown that the sense of familiarity and the sense of self are processed independently in normal participants [9,11]. First, a left-hand advantage is observed only in recognizing self faces, not familiar faces, suggesting that the processing of self is dominated in the right hemisphere [4-6]. Second, functional magnetic resonance imaging studies have revealed that different cortical regions are 
recruited to process self faces and familiar faces respectively [21]. For example, regions such as the superior frontal gyrus, medial frontal gyrus, and inferior parietal lobule in the right hemisphere are selectively activated in processing self faces (versus familiar faces), whereas familiar faces selectively activate bilateral temporoparietal cortical regions [8-11,22-25]. Therefore, the two component processes are dissociable in the normal population, suggesting that they can be selectively impaired in self-face recognition in schizophrenia.

In this study, we directly investigated which component process of self-face recognition, the sense of familiarity or the sense of self, was impaired in schizophrenia. Three types of faces were included: self faces, faces of famous movie stars (i.e., familiar faces), and faces of strangers (novel faces). Because patients with schizophrenia also have difficulties in general cognitive abilities [26,27], absolute measures of their ability to recognize faces, such as accuracy and response time, might be confounded with the general deficit. Here we measured the bias in recognizing the identity of a morphed face created by blending face features between any of two identities from the self face, the familiar face, and the novel face. If the sense of self is impaired, we predict that patients, relative to normal participants, would be more likely to classify self-familiar morphed faces as familiar faces. In contrast, if the sense of familiarity is impaired, patients shall tend to judge familiar-novel morphed faces as novel faces.

\section{Materials and methods}

\subsection{Participants}

Twenty-four patients with schizophrenia (18 males and 6 females) participated in this study. They were recruited from Beijing Anding Hospital, Capital Medical University, China. The patients were diagnosed according to the DSMIV by two experienced psychiatrists following a comprehensive evaluation including psychiatric examination, proxy interview, and record review. Ten patients were diagnosed as the paranoid type, and 14 were diagnosed as the undifferentiated type. The patients had no history of electroconvulsive therapy, and had no substance abuse, mental retardation, neurological injury or disease, or medical diseases that may affect brain functions. The patients had a mean duration of illness of 5.0 years $(\mathrm{SD}=3.6)$. At the time of testing, four patients were drug-naïve, and 20 were taking atypical antipsychotic drugs (clozapine, risperidone, quetiapine, or olanzapine).

Twenty-three normal participants (11 males and 12 females) were recruited as controls with matched average years of education. Normal participants had no history of DSM-IV Axis I or II disorders and had no family history of psychotic illnesses.

All schizophrenic and normal participants were righthanded, with normal or corrected-to-normal vision. Table 1 presents detailed demographic information. The study was approved by the Institutional Review Board of Beijing Normal University and of Beijing Anding Hospital of Capital Medical University. Prior to the testing, written informed consent was obtained from all participants.

\subsection{Stimuli}

There were three face images for each participant: her/his own face (the self face), the face of a famous movie star who was able to be recognized by the participant at first sight (the familiar face), and the face of another participant who s/he had never met (the novel face). Of note, faces of movie stars, not faces of participants' relatives, were chosen as the familiar faces, simply because the representation of relatives' faces may be associated with that of self faces for Chinese subjects [28]. All images are the frontal views of faces with an external contour (a roughly oval shape with hair on the top and sides) cropped. The size of face images was $350 \times 350$ pixels $\left(10.4^{\circ} \times 10.4^{\circ}\right.$ visual angle at a distance of $60 \mathrm{~cm}$ ). The self face was mirror-transposed, and the gender of the familiar and novel faces was matched to that of the self face. The expression of all faces was neutral.

Morphed faces were generated by photo-morphing software (FantaMorph). The software gradually blended facial features between any two of the three types of faces using 40-80 anatomical landmarks (e.g., points on eyes and nose) that were manually aligned for each face pair. The morphing step was $5 \%$, thus resulting in a series of 19 morphed faces plus two original faces (Figure 1(a)). Note that the faces shown here are not those of the participants but those of two volunteers who have seen this manuscript and figure,

Table 1 Demographic information of patients and controls ${ }^{\text {a) }}$

\begin{tabular}{llll}
\hline & Patients $(n=24)$ & Control $(n=23)$ \\
\hline Age & $27.1(6.6)$ & $23.0(4.0)$ \\
Education (years) & $12.4(2.2)$ & $13.7(3.1)$ \\
Gender (M/F) & $18 / 6$ & \\
PANSS & $64(12.5)$ & - \\
Drug dosage (chlorpromazine equivalent) & $409.7(231.6)$ & - \\
\hline
\end{tabular}

a) Values are given as mean (SD); PANSS, Positive and Negative Syndrome Scale. 
(a)

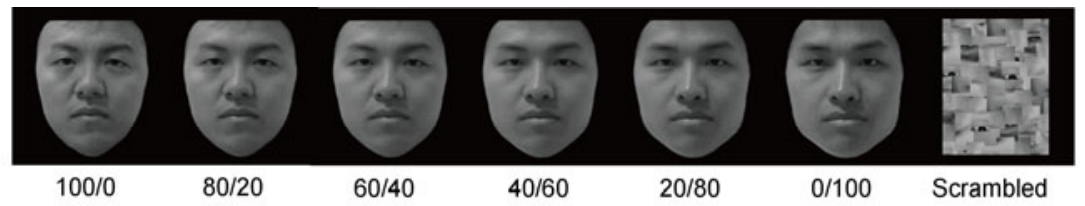

(b)
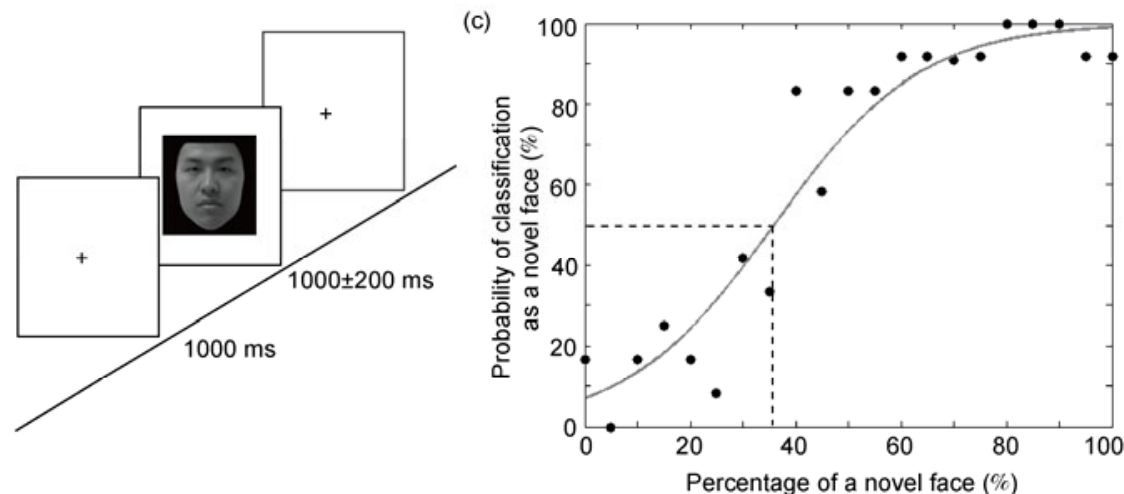

Figure 1 Experimental procedure and data analysis. (a) Exemplar stimuli of a self-novel pair. Morphed faces were created by blending facial features of a self face (left) and a novel face (right) at steps of 5\%. Numbers below each image indicate the percentage contributions from the self face and the novel face respectively. Mosaic-like images were created by scrambling either the self face or novel face. (b) Identification task. A morphed face was presented for $1 \mathrm{~s}$, followed by a fixation for $1 \mathrm{~s}$ with a jitter of $200 \mathrm{~ms}$. Participants were required to categorize morphed faces according to the identities of the original faces. (c) Results for a typical patient. Each dot represents the patient's judgment of morphed faces at a different level of morphing. The curve is the fitted line from a logistic psychometric function based on the participants' raw response. The dotted line indicates the percentage of the novel face at the psychological switching point (i.e., $50 \%$ probability).

and have provided written consent for publication. Additionally, original faces were scrambled into mosaic-like images (scrambled faces) for catch trials. Four scrambled faces were created for each series. Thus, for each participant, there were three series of morphed faces (i.e., self-familiar, self-novel, and familiar-novel), each of which contained 21 faces and four scrambled faces.

\subsection{Experimental procedure}

The experiment consisted of three blocks-the self-familiar block, the self-novel block, and the familiar-novel block, the order of which were counterbalanced across participants. Within a block, images were presented sequentially at the center of the screen in a pseudorandom order. Each morphed face and each scrambled face was repeated eight times within a block, totaling 200 trials per block. In a trial, an image was presented for $1 \mathrm{~s}$, followed by a central fixation for $1 \mathrm{~s}$ with a jitter of $200 \mathrm{~ms}$ (Figure 1(b)). Participants were instructed to make a two-alternative forced-choice (2AFC) to indicate whether the morphed face was more similar to one or the other possible identity. For example, in the self-familiar block, participants were instructed to decide whether a morphed face was more similar to the self face or the face of a movie star. Choices were made by pressing one of two buttons with one hand. Trials with scrambled faces served as catch trials, and participants were instructed to press a button with the other hand when they observed a scrambled face. The button press was counterbalanced between hands within participants. For example, in one half of the blocks, the left hand was assigned to press a button in response to morphed faces and the right hand pressed a button for reporting the presence of scrambled faces. The assignment of hands in pressing buttons was switched in the other half of the blocks. All participants were seated $60 \mathrm{~cm}$ from the screen and finished all three blocks.

\subsection{Data analysis}

Trials with no response or more than one response were excluded (3.4\% of all trials), and the data for the familiar-novel block of one patient was also excluded because the correct response to the catch trials $(37.5 \%)$ was not significantly higher than random guessing. In addition, the data for the self-familiar block of two patients and the data for the self-novel block of a control participant were unavailable owing to computer hardware failure.

To locate the categorical boundary of participants' responses to morphed faces, a logistic psychometric function with a maximum likelihood criterion was used to fit the data for each face pair and for each participant. The categorical boundary, or the psychological switch point, was the point where participants had equal responses to both identities. The percentage of morphing at the switching point was then defined as the bias for each face pair and for each participant (Figure 1(c)). The bias served as a measure of ability in recognizing one type of face relative to the other.

Specifically, the bias in the self-familiar block was defined as the percentage of the familiar face (versus the self 
face) in the self-familiar morphed face at the switching point, with a larger value indicating a higher tendency of identifying the morphed face as the self face. Because the self face and the familiar face were both familiar to participants, the value of the bias was mainly affected by the sense of self. Therefore, the bias acquired in the self-familiar block was a measure of the sense of self (i.e., the self bias). Similarly, the bias in the familiar-novel block was defined as the percentage of the novel face (versus the familiar face) in the familiar-novel morphed face at the switching point, with a larger value indicating a higher tendency of classifying the morphed face as the familiar face. Because the familiar face and the novel face mainly differed in familiarity, the bias in the familiar-novel block provided an index of the sense of familiarity (i.e., the familiarity bias). Finally, the bias in the self-novel block was defined as the percentage of the novel face (versus the self face) in the self-novel morphed face at the switching point, with a larger value indicating a higher tendency of identifying the morphed face as the self face. Relative to the novel face, the self face was both familiar and self-related; therefore, the bias in the selfnovel block was affected by both the sense of familiarity and the sense of self, thus providing a measure of a general ability in recognizing the self face (i.e., the self-recognition bias).

Ideally, if there were no bias toward to either identity of morphed faces, the value should be $50 \%$. However, regardless of how carefully face stimuli were matched, low-level differences across stimuli remained. Therefore, instead of comparing patients' biases to the absolute value of $50 \%$, we compared them with normal participants' biases when they performed the same task. In other words, we used normal participants' biases as the baseline to evaluate patients' biases.

Outlier biases (i.e., 1.5 IQRs above the third quartile or 1.5 IQRs below the first quartile; IQR: the interquartile range) were excluded from further analyses (4.8\% of the data). Statistical analyses were Pearson's correlation analyses, repeated-measure analyses of variance (ANOVAs), and independent two-sample $t$-tests.

\section{Results}

To examine whether self-face recognition in patients with schizophrenia was independently modulated by the sense of self and the sense of familiarity, correlations between biases were calculated. Figure 2(a) shows the results of correlation analyses. As expected, correlations were found between the self bias and self-recognition bias $(r=0.58, P=0.005)$, and between the familiarity bias and self-recognition bias $(r=$ $0.40, P=0.058)$, indicating that both component processes, the sense of self and the sense of familiarity, contributed to the recognition of self faces. Importantly, no significant correlation was observed between the self bias and familiarity
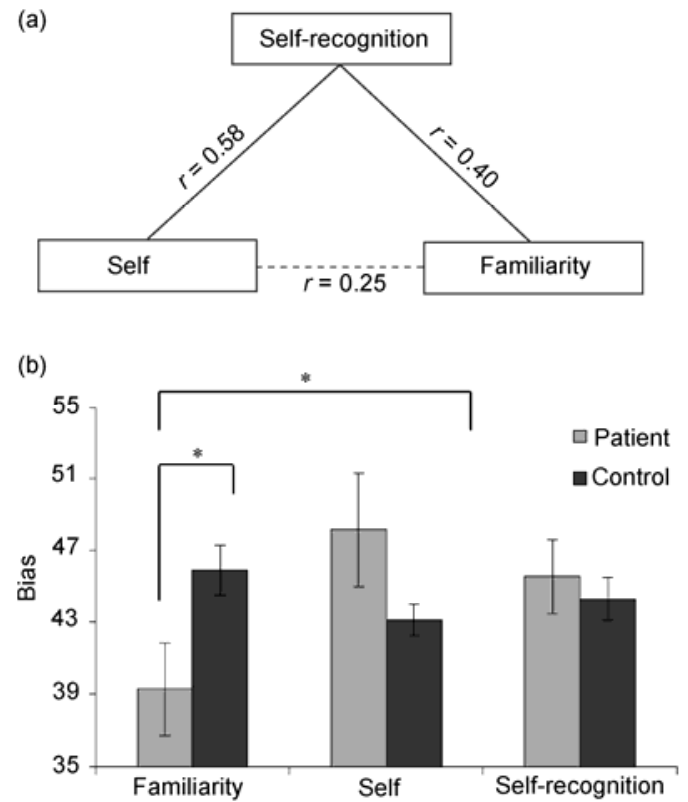

Figure 2 The deficit in the sense of familiarity in self-face recognition. (a) Correlations among the sense of self, the sense of familiarity, and self-face recognition. Both component processes were correlated with self-face recognition, while they were dissociated from each other. (b) Behavioral performance of patients and normal participant (i.e., controls). The patients were deficient in recognizing the familiarity of self faces as compared with the controls, and there was no significant difference in the sense of self between groups. The $Y$ axis indicates the magnitude of the bias. $* P<0.05$.

bias ( $r=0.25, P=0.246)$, suggesting that although selfface recognition was modulated by both the sense of self and the sense of familiarity, the two component processes were dissociable. Next, we examined which component process of self-face recognition was selectively impaired in schizophrenia.

To this end, we compared the behavioral performance of the patients with that of the normal participants. A two-way ANOVA with participant group (patient versus normal) by type of bias (self versus familiarity versus self-recognition) showed a significant interaction $(F(2,70)=4.98, P=0.01)$ (Figure 2(b)). Post-hoc two-sample $t$-tests further revealed that the familiarity bias observed in the patients was significantly smaller than that for the normal participants $(t(41)=$ 2.16, $P=0.04)$, suggesting that the patients were deficient in recognizing the familiarity of faces. In contrast, there was no significant difference between two groups in the self bias $(t(40)=1.44, P=0.16)$ and in the self-face recognition bias $(t(42)=0.52, P=0.60)$. This observation was further confirmed by a two-way interaction of the participant group (patient versus normal) by type of bias (self versus familiarity) $(F(1,37)=6.97, P=0.01)$.

\section{Discussion}

In this study, we directly tested which component process of self-face recognition, the sense of familiarity or the sense of 
self, was impaired in schizophrenia. In accord with previous findings for the normal population $[9,11]$, we found that the sense of familiarity and the sense of self were processed independently in schizophrenia as well. More importantly, it is the sense of familiarity, not the sense of self, that was selectively impaired in self-face recognition in schizophrenia. Taken together, our study provides evidence against the hypothesis that the deficit observed in self-face recognition in schizophrenia arises from the breakdown of self-awareness.

The dissociation in recognizing the self and the familiarity of self faces in schizophrenia is consistent with previous findings for the normal population $[9,11]$. Note that the lack of correlation between these two component processes in the patients cannot be accounted for by insufficient statistical power or differences in stimuli, because both processes were correlated with self-face recognition. In addition, our finding is consistent with previous studies reporting that patients with schizophrenia are deficient in recognizing the familiarity of faces [19,29-32]. On the other hand, no deficit in the sense of self in self-face recognition was found for the patients tested. Instead, the patients, relative to the normal participants, tended to identify self-familiar morphed faces as the self face, implying that the sense of self in schizophrenia may not be diminished, but rather exaggerated [20]. Taken together, our study suggests that the poor performance in recognizing self faces arises from impairment in the sense of familiarity, not the sense of self.

Several important issues remain. First, our finding does not argue against the idea that the alteration of self is a core deficit of schizophrenia [13,33-39]. Instead, we argue that the deficit in recognizing self faces in schizophrenia may be not associated with the alteration of the self. Experimental paradigms that measure the hemispheric asymmetry in self processing $[13,16]$, for example, may provide a better way of characterizing how the concept of self is represented in schizophrenia. Second, a previous study with a similar paradigm failed to find any deficit in processing either the familiarity or the self of self faces in schizophrenia [13], which is inconsistent with our finding. One possible reason for the different findings is that in our study faces were morphed at a finer scale (5\% versus $12.5 \%$ in Kircher et al.'s study [13]), and the bias derived from the fitting of a logistic psychometric function was used to index the patients' behavioral performance in recognizing self faces. Therefore, our study may provide a more sensitive measure of the self-face recognition in schizophrenia. Third, schizophrenia is a spectrum of disorders, and thus highly heterogeneous and sophisticated. It would be interesting to examine whether different types of schizophrenia show distinct patterns of deficits in self-face recognition by first classifying patients into relatively homogeneous sub-groups.

We thank Yiying Song, Jingguang Li, and Ruosi Wang for comments on the manuscript. This work was supported by the 100 Talents Program of the Chinese Academy of Sciences, the National Natural Science Foundation of
China (91132703), the National Basic Research Program of China (2010CB833903, 2011CB505402), the China Postdoctoral Science Foundation (20060400367), and the Fundamental Research Funds for Central Universities. The funding agencies had no role in study design; in the collection, analysis and interpretation of data; in the writing of the report; and in the decision to submit the paper for publication. All authors declare no conflicts of interest.

1 Gallup G G. Chimpanzees: Self-recognition. Science, 1970, 167: 86-87

2 Parker S T, Mitchell R W, Boccia M L. Self-awareness in Animals and Humans: Developmental Perspectives. Cambridge: Cambridge University Press, 1994

3 Povinelli D J, Gallup G G, Eddy T J, et al. Chimpanzees recognize themselves in mirrors. Anim Behav, 1997, 53: 1083-1088

4 Keenan J P, McCutcheon B, Freund S, et al. Left hand advantage in a self-face recognition task. Neuropsychologia, 1999, 37: 1421-1425

5 Keenan J P, Freund S, Hamilton R H, et al. Hand response differences in a self-face identification task. Neuropsychologia, 2000, 38: 1047-1053

6 Keenan J P, Ganis G, Freund S, et al. Self-face identification is increased with left hand responses. Laterality, 2000, 5: 259-268

7 Tong F, Nakayama K. Robust representations for faces: Evidence from visual search. J Exp Psychol Hum Percept Perform, 1999, 25: 1016-1035

8 Platek S M, Keenan J P, Gallup G G, et al. Where am I? The neurological correlates of self and other. Cogn Brain Res, 2004, 19: 114122

9 Platek S M, Loughead J, Gur R C, et al. Neural substrates for functionally discriminating self-face from personally familiar faces. Hum Brain Mapp, 2006, 27: 91-98

10 Uddin L Q, Kaplan J T, Molnar-Szakacs I, et al. Self-face recognition activates a frontoparietal "mirror" network in the right hemisphere: An event-related fMRI study. NeuroImage, 2005, 25: 926-935

11 Sugiura M, Watanabe J, Maeda Y, et al. Cortical mechanisms of visual self-recognition. NeuroImage, 2005, 24: 143-149

12 Platek S M, Wathne K, Tierney N G, et al. Neural correlates of self-face recognition: An effect-location meta-analysis. Brain Res, 2008, 1232: 173-184

13 Kircher T T, Seiferth N Y, Plewnia C, et al. Self-face recognition in schizophrenia. Schizophr Res, 2007, 94: 264-272

14 Irani F, Platek S M, Panyavin I S, et al. Self-face recognition and theory of mind in patients with schizophrenia and first-degree relatives. Schizophr Res, 2006, 88: 151-160

15 Platek S M, Gallup G G. Self-face recognition is affected by schizotypal personality traits. Schizophr Res, 2002, 57: 81-85

16 Platek S M, Myers T E, Critton S R, et al. A left-hand advantage for self-description: The impact of schizotypal personality traits. Schizophr Res, 2003, 65: 147-151

17 Troje N, Kersten D. Viewpoint-dependent recognition of familiar faces. Perception, 1999, 28: 483-488

18 Gallup Jr G G. Self-awareness and the emergence of mind in primates. Am J Primatol, 1982, 2: 237-248

19 Caharel S, Bernard C, Thibaut F, et al. The effects of familiarity and emotional expression on face processing examined by ERPs in patients with schizophrenia. Schizophr Res, 2007, 95: 186-196

20 Lee J, Kwon J S, Shin Y W, et al. Visual self-recognition in patients with schizophrenia. Schizophr Res, 2007, 94: 215-220

21 Keenan J, Wheeler M, Gallup G, et al. Self-recognition and the right prefrontal cortex. Trends Cogn Sci, 2000, 4: 338-344

22 Decety J, Chaminade T. When the self represents the other: A new cognitive neuroscience view on psychological identification. Conscious Cogn, 2003, 12: 577-596

23 Morita T, Itakura S, Saito D N, et al. The role of the right prefrontal cortex in self-evaluation of the face: A functional magnetic resonance imaging study. J Cogn Neurosci, 2008, 20: 342-355

24 Keenan J P, Nelson A, O'Connor M, et al. Neurology: Self-recognition and the right hemisphere. Nature, 2001, 409: 305 
25 Sugiura M, Sassa Y, Jeong H, et al. Face-specific and domain-general characteristics of cortical responses during self-recognition. NeuroImage, 2008, 42: 414-422

26 Anilkumar A P P, Kumari V, Mehrotra R, et al. An fMRI study of face encoding and recognition in first-episode schizophrenia. Acta Neuropsychiatr, 2008, 20: 129-138

27 Williams L M, Loughland C M, Gordon E, et al. Visual scanpaths in schizophrenia: Is there a deficit in face recognition? Schizophr Res, 1999, 40: 189-199

28 Zhu Y, Qi J, Zhang J. Self-face identification in Chinese students. Acta Psychol Sin, 2004, 36: 442-447

29 Archer J, Hay D C, Young A W. Movement, face processing and schizophrenia: Evidence of a differential deficit in expression analysis. Br J Clin Psychol, 1994, 33: 517-528

30 Baudouin J Y, Martin F, Tiberghien G, et al. Selective attention to facial emotion and identity in schizophrenia. Neuropsychologia, 2002, 40: 503-511

31 Hooker C, Park S. Emotion processing and its relationship to social functioning in schizophrenia patients. Psychiatry Res, 2002, 112: 41-50
32 Salem J E, Kring A M, Kerr S L. More evidence for generalized poor performance in facial emotion perception in schizophrenia. J Abnorm Psychol, 1996, 105: 480-483

33 Hecht D. Schizophrenia, the sense of 'self' and the right cerebral hemisphere. Med Hypotheses, 2010, 74: 186-188

34 Lindner A, Thier P, Kircher T T, et al. Disorders of agency in schizophrenia correlate with an inability to compensate for the sensory consequences of actions. Curr Biol, 2005, 15: 1119-1124

35 Kircher T T, David A S. The Self in Neuroscience and Psychiatry. Cambridge: Cambridge University Press, 2003

36 Kircher T T, Leube D T. Self-consciousness, self-agency, and schizophrenia. Conscious Cogn, 2003, 12: 656-669

37 Knoblich G, Stottmeister F, Kircher T T. Self-monitoring in patients with schizophrenia. Psychol Med, 2004, 34: 1561-1569

38 Pauly K, Kircher T, Weber J, et al. Self-concept, emotion and memory performance in schizophrenia. Psychiatry Res, 2010, 186: $11-17$

39 Sass L A, Parnas J. Schizophrenia, consciousness, and the self. Schizophr Bull, 2003, 29: 427

Open Access This article is distributed under the terms of the Creative Commons Attribution License which permits any use, distribution, and reproduction in any medium, provided the original author(s) and source are credited. 